\title{
A randomised controlled trial investigating the analgesic efficacy of transversus abdominis plane block for adult laparoscopic appendicectomy
}

\author{
Darell Alexander Tupper-Carey ${ }^{1}$, FRCA, Shahridan Mohd $\underline{\text { Fathill}}^{1}$, FRCA, Yin Kiat Glenn $\underline{\text { Tan }}^{1}$, FANZCA, Yuk Man Kan ${ }^{2}$, FRCS,
} Chern Yuen $\underline{\text { Cheong }}^{2}$, Mrcs, Fahad Javaid Siddiqui ${ }^{3,4}$, PhD, Pryseley Nkouibert $\underline{\text { Assam }}^{3,4}$, PhD

INTRODUCTION We conducted a single-centre, prospective randomised clinical trial to investigate the analgesic efficacy of transversus abdominis plane (TAP) block in adult patients undergoing laparoscopic appendicectomy.

METHODS Patients undergoing urgent laparoscopic appendicectomy under general anaesthesia alone (control group) and general anaesthesia supplemented by TAP block (TAP intervention group) were compared. All patients received a multimodal analgesia regime, which included postoperative morphine via a patient-controlled analgesia device. The primary endpoints were morphine consumption at 12 hours and 24 hours postoperatively. Secondary endpoints included pain scores, incidence of nausea and vomiting, and time to hospital discharge. A total of 58 patients were recruited, with 29 patients in each group.

RESULTS Mean postoperative morphine consumption at 12 hours (control group: $11.45 \pm 7.64$ mg, TAP intervention group: $9.79 \pm 8.09 \mathrm{mg} ; \mathrm{p}=0.4264$ ) and 24 hours (control group: $13.38 \pm 8.72 \mathrm{mg}$, TAP intervention group: $11.31 \pm 8.66 \mathrm{mg}$ $p=0.3686$ ) for the control and TAP intervention groups were not statistically different. Secondary outcomes were also not different between the two groups. Length of stay in the post-anaesthesia care unit was significantly shorter for the TAP intervention group, with a trend toward faster hospital discharge being observed.

CONCLUSION TAP block, a regional anaesthetic procedure performed immediately prior to skin incision for laparoscopic appendicectomy, did not significantly improve postoperative analgesia outcomes.

Keywords: laparoscopic appendicectomy, postoperative analgesia, regional anaesthesia, transversus abdominis plane block

\section{INTRODUCTION}

The pioneering gynaecologist, Dr Kurt Semm, performed the first laparoscopic appendicectomy in 1981. ${ }^{(1)}$ This procedure has subsequently been adopted by an increasing proportion of general surgeons as the surgical technique of choice for appendicectomy. Surgery is required for acute appendicitis to prevent perforation, as well as to manage complications that may occur with this disease. The laparoscopic technique is favoured over the traditional open method of appendicectomy, as the former is associated with a lower incidence of postoperative surgical complications and faster recovery to normal daily activities. ${ }^{(2,3)}$ Despite the use of minimally invasive surgery, patients may require hospitalisation for over 24 hours following laparoscopic appendicectomy, and postoperative pain is a common cause for extended length of hospital stay. ${ }^{(4)}$

The transversus abdominis plane (TAP) block is a regional anaesthetic procedure wherein local anaesthetic solution is injected into the TAP of the abdominal wall. Recently, this procedure has gained popularity, as it was shown to reduce both postoperative pain and opiate analgesic requirements following a number of different abdominal surgical procedures. ${ }^{(5-9)}$ The aim of this study was to investigate the postoperative analgesic effects of TAP block as a supplement to a general anaesthetic for laparoscopic appendicectomy in adult patients. A literature search did not identify any previous studies investigating the use of TAP block in adult patients undergoing this common surgical procedure.

\section{METHODS}

The study was performed at Alexandra Hospital, Singapore, from December 2012 to December 2013 and adhered to the relevant national biomedical research regulations, including approval from the research ethics committee and informed consent from all patients. Study design consisted of a prospective randomised controlled trial of patients undergoing laparoscopic appendicectomy that compared the addition of TAP block to a standardised general anaesthetic and surgical technique. Anaesthetic and postoperative pain management was standardised for all patients with the aim of minimising differences between the two groups except for the TAP block intervention.

The primary endpoints for the study were absolute and equianalgesic morphine consumption values at 12 hours and 24 hours postoperatively. Secondary endpoints were: (a) pain scores (at rest and on movement) during the first 24 hours following surgery; (b) incidence of nausea and vomiting during the first 24 hours following surgery; and (c) time to hospital discharge.

Enrolment of patients into the study was done while the patients were awaiting urgent surgery in the hospital's surgical

${ }^{1}$ Department of Anaesthesia, ${ }^{2}$ Department of Surgery, Ng Teng Fong General Hospital, JurongHealth, ${ }^{3}$ Centre for Quantitative Medicine, Duke-NUS Medical School, ${ }^{4}$ Singapore Clinical Research Institute, Singapore

Correspondence: Dr Darell Alexander Tupper-Carey, Senior Consultant, Department of Anaesthesia, Ng Teng Fong General Hospital, JurongHealth, 1 Jurong East Street 21, Singapore 609606. tupper_carey_darell@juronghealth.com.sg 
ward. Patients were included in the study if they were over 21 years of age and undergoing urgent surgery for acute appendicitis. Exclusion criteria were: (a) previous surgery within the last six weeks; (b) preoperative medical history of chronic pain or opiate analgesic medication; (c) inability to understand instructions regarding numeric rating scale (NRS) pain assessment or the use of patient-controlled analgesia (PCA) machine; (d) pregnancy; (e) known allergy to local anaesthetic drugs; and (f) weight less than $50 \mathrm{~kg}$.

Following emergency admission to the hospital, the patients were resuscitated with intravenous fluids, antibiotics and analgesics according to the standard hospital clinical practice within the emergency and surgical departments. Resuscitation prior to surgery was directed by the on-duty surgical team that was responsible for the patient. On arrival in the holding bay of the operation theatre, the patients were randomised to the TAP intervention or control groups by a study investigator. For patients randomised to the TAP intervention group, general anaesthesia was supplemented by a TAP block. Those in the control group underwent urgent laparoscopic appendicectomy under general anaesthesia alone.

A standardised general anaesthetic technique was used for all the patients and included rapid sequence induction of anaesthesia (propofol 1-3 mg/kg; suxamethonium 1-1.5 mg/kg), and tracheal intubation and mechanical ventilation with desflurane in an oxygen-enriched air mixture. Atracurium was administered for intraoperative neuromuscular paralysis followed by neuromuscular reversal, as guided by the anaesthetist. Intraoperative analgesia consisted of fentanyl (1-2 mcg/ $\mathrm{kg}$ ) prior to skin incision with further bolus doses $(1 \mathrm{mcg} / \mathrm{kg})$, as judged necessary by the anaesthetist responsible for the patient. Ondansetron was administered (4 mg intravenously) to patients with previous history of postoperative nausea and vomiting.

Patients in the TAP intervention group received 20-mL

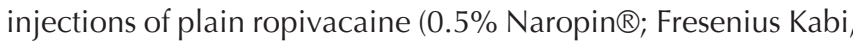
IL, USA) to each side of the abdominal wall. The TAP block intervention, which was performed by three study investigators who were anaesthetists with experience in regional anaesthesia, was done following the induction of anaesthesia and prior to surgical skin incision. The intervention was performed using aseptic technique with ultrasonography guidance (Logic $\mathrm{E} \circledast$; $\mathrm{GE}$, Buckinghamshire, UK) using an in-plane technique for needle tip guidance (Stimuplex® A; B Braun, Melsungan, Germany) through the abdominal wall into the neurovascular fascial layer separating the internal oblique and transversus abdominis muscles. The injection was directed by identifying the three muscle layers lateral to the rectus muscle and using a standard injection technique in the mid-axillary line at the midpoint between the inferior costal margin and iliac crest. ${ }^{(10)}$ Prior to surgery, a 10-mL syringe prepared by the study investigator was given to the surgeon for infiltration of the laparoscopic port sites; the syringes contained $0.5 \%$ plain bupivacaine for the control group and $0.9 \%$ normal saline for the TAP intervention group.

Following emergence from anaesthesia, tracheal extubation was performed and patients were transferred to the postanaesthesia care unit (PACU). The surgical technique was standardised for all patients and included laparoscopic insufflation through an umbilical port, with two additional ports placed in the left and right lower quadrants. After transfer to the PACU, the patients were monitored by the recovery nursing staff and assessed for fitness for discharge every 15 minutes using standard discharge criteria. The fitness for discharge criteria included a pain score $\leq 4$ at rest and the absence of significant nausea and vomiting. An intravenous morphine PCA system, which was programmed to a standard regime (1-mg bolus, five-minute lockout, maximum hourly dose of $5 \mathrm{mg}$ and no background infusion), was administered to each patient. All patients were instructed by the PACU nursing staff on how to use the PCA to self-administer bolus doses, with the aim of achieving a pain score $\leq 4$ at rest or on movement. ${ }^{(11)}$ Regular oral analgesics (etoricoxib 90-120 mg once daily based on weight and paracetamol $1 \mathrm{~g}$ six hourly) were also prescribed for all patients, unless contraindicated. Ondansetron (4-8 mg intravenously) was prescribed eight hourly, as required for treatment of nausea and vomiting.

Morphine PCA was continued for a minimum of 12 hours postoperatively for all patients; after 12 hours post-surgery, a member of the on-duty anaesthetic pain team visited all patients to assess for morphine PCA discontinuation based on predefined criteria: pain score $\leq 4$ at rest and on movement, and improved clinical condition. On discontinuing morphine PCA, additional rescue analgesia was prescribed, when required, to be administered if the patient reported a pain score $>4$ (oral morphine elixir $5 \mathrm{mg}$ prescribed as required six hourly). Morphine PCA was continued if pain scores were $>4$ or if the pain team decided that continuation was necessary on clinical grounds. Thereafter, the pain team reviewed patients twice daily until morphine PCA was discontinued. Following discontinuation of morphine PCA, the patients were discharged from follow-up with the acute pain team.

On discharge from the PACU, the patients were transferred to the surgical ward and managed by the operating surgical team. Postoperative management included antibiotic therapy and intravenous fluids. Oral fluids and feeding were encouraged for all patients, unless specified by the surgeon. Patients were mobilised as soon as permissible following surgery, with the aid of the ward nursing staff. Assessment of suitability for hospital discharge was performed in the morning and afternoon on each postoperative day by the surgical team responsible for the patient.

The study period commenced on induction of anaesthesia in the operation theatre and continued for 48 hours following surgery or until discharge from hospital (if the latter occurred within 48 hours following surgery). Routine data collection ceased at the end of the study period, and patients who remained in hospital for longer than 48 hours following surgery were reviewed daily by a study investigator to monitor for adverse events until hospital discharge. The study was considered completed for each patient at the time of discharge from hospital.

Postoperative pain was assessed using the NRS system, which was self-reported by patients as a standard assessment of pain severity on a scale from $0-10$, both at rest and on movement. Measurements of pain and morphine consumption 
were recorded by the nursing staff according to standard hospital clinical guidelines for postoperative pain management and obtained repeatedly over time for a maximum period of 48 hours following surgery. The presence of either nausea or vomiting was recorded simultaneously with pain scores during the study period using a standard hospital clinical scoring system. All postoperative study measurements were taken from the time of admission to the PACU, unless otherwise stated. The grade of appendicitis was recorded for each patient based on the findings at the initial laparoscopy and classified using the following scale: normal, inflamed, inflamed with complications, or perforated with peritonitis. The time course of each patient's hospital visit, from the time of admission (including other relevant time points during the hospital stay) to the time of hospital discharge, was also recorded.

During the study period, the following parameters were recorded following surgery: (a) pain scores at rest and on movement (at 0, 1, 2, 4, 8, 12, 24, 36 and 48 hours); (b) PCA morphine consumption at 12 hours; (c) equianalgesic morphine consumption (morphine elixir was converted to equianalgesic values of intravenous morphine on a 3:1 ratio) at 24 hours; (d) requirement for rescue analgesia (number of doses and total dose of oral morphine) or extended morphine PCA administration ( $>18$ hours); (e) number of successful and unsuccessful PCA bolus attempts (a high proportion of failed bolus doses was defined as $>25 \%$ of unsuccessful bolus attempts); and ( $\mathrm{f}$ ) the presence of nausea or vomiting for each 12-hour time period during the first 48 hours following surgery. In addition, time of assessment for fitness for discharge and the actual time of hospital discharge were recorded. We also recorded adverse events, including potential complications from the TAP intervention (e.g. local anaesthetic systemic toxicity or complications arising from the needle injection).

Patients were randomised in a 1:1 allocation ratio using a computer-generated random number sequence and allocated to either the TAP intervention or control group using serially numbered opaque sealed envelopes. The study investigator performed the randomisation immediately prior to the study intervention; apart from the intervention, this investigator was not involved in the anaesthetic or surgical care of the patients. The anaesthetic and surgical teams were blinded to the allocation of patients, as they were asked to leave the operation theatre for 15-20 minutes (approximately the time required to perform a TAP block) while the study investigator was performing the TAP block for the intervention group or no intervention for the control group. The syringes prepared by the study investigator, which contained either local anaesthetic drug solution or saline for infiltration of the laparoscopic port site tissues, were drawn up so that the surgeon was unaware of its contents.

The required sample size of 58 patients (29 in each group) was based on the following assumptions: 1:1 allocation ratio; $5 \%$ Type I error; and $80 \%$ power of detecting a mean difference in morphine consumption of $6 \mathrm{mg}$ between the two groups over the first 24 hours $(6 \mathrm{mg}$ for the TAP intervention group and $12 \mathrm{mg}$ for the control group; standard deviation [SD] $8 \mathrm{mg}$ ) based on a two-sample $t$-test.

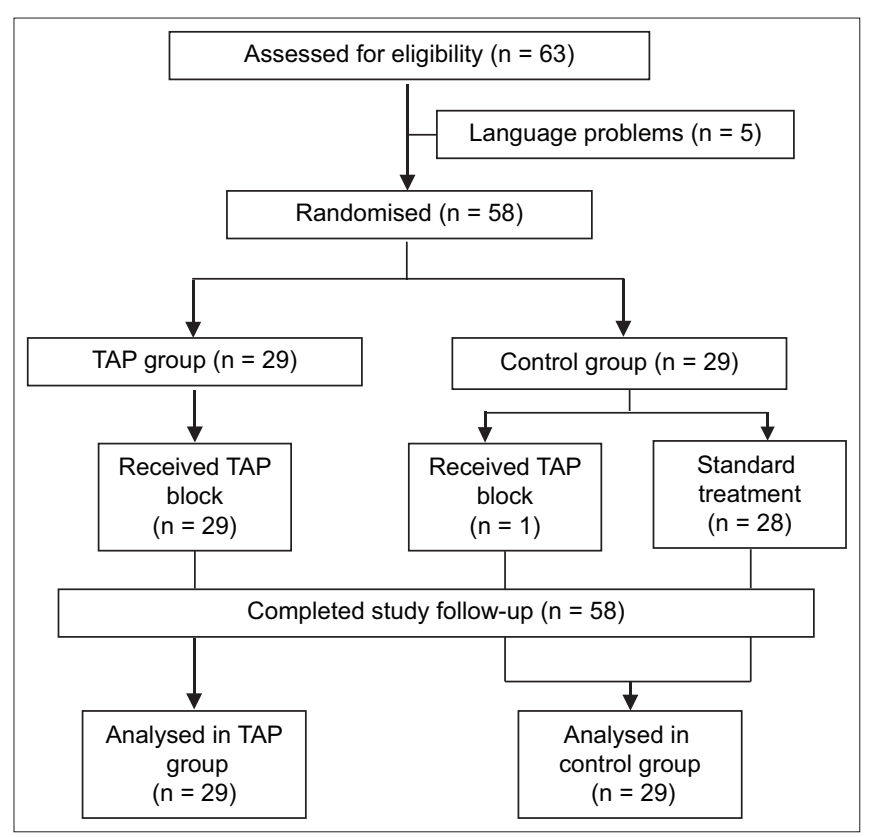

Fig. 1 Flowchart for patient participation for the study. TAP: transversus abdominis plane

All analyses were performed based on the intention-to-treat principle. Data was summarised according to patient groups using either mean $\pm \mathrm{SD}$ or median (interquartile range) for continuous variables, as appropriate, and frequency (proportions) for categorical variables. The groups were compared using twosample $t$-test and Mann-Whitney $U$ test for continuous variables, and Fisher's exact test for categorical variables. Repeated measures analysis of variance was used to compare pain scores that were assessed repeatedly over time between the patient groups. Significance level was set at $5 \%$ and all tests were twosided. The analysis of data was performed using SAS software version 9.2 (SAS Institute, Cary, NC, USA).

\section{RESULTS}

A total of 58 patients were enrolled in the study, with 29 patients randomised to each group. All patients completed the 48-hour study period and were followed up until discharge from hospital (Fig. 1). For two patients in the control group, laparoscopic appendicectomy was converted to open surgical procedure; both patients were included in the analysis on an intention-to-treat basis. One patient, who was randomised to the control group but incorrectly received a TAP block, was also analysed in the control group in accordance with the intention-to-treat principle. Table I shows the demographic and surgical characteristics of the two patient groups. A higher proportion of women were allocated to the TAP intervention group compared to the control group, which was reflected in the lower median values of height and weight recorded for the TAP intervention group. Otherwise, the two groups were comparable with respect to the various parameters assessed. The two groups were also similar in terms of the grade of appendicitis, as well as other clinical parameters that may have influenced postoperative pain and recovery.

Table II shows the analgesia drug requirements for the two patient groups during the study period. For the primary endpoints 
(PCA morphine consumption at 12 hours and equianalgesic morphine consumption at 24 hours), mean values were not significantly different between the two groups. Similarly, other indicators of postoperative analgesia efficacy, including the number of unsuccessful PCA bolus attempts and requirement for oral rescue analgesia, were also not significantly different. Also shown in Table II is supplementary data (such as intraoperative fentanyl requirements, and nausea and vomiting scores during the first 24 hours following surgery). Intraoperative fentanyl requirements were significantly lower in the TAP group $(p=0.0058)$. Nausea and vomiting scores were not significantly different between the two groups, although there was a trend toward higher values in the TAP group.

Table I. Baseline characteristics of the patients according to patient group.

\begin{tabular}{|c|c|c|}
\hline \multirow[t]{2}{*}{ Variable } & \multicolumn{2}{|c|}{ No. (\%) } \\
\hline & Control $(n=29)$ & $\operatorname{TAP}(n=29)$ \\
\hline Female gender & $4(13.8)$ & $8(27.6)$ \\
\hline Age* $^{*}(y r)$ & $29(27-37)$ & $27(26-36)$ \\
\hline Weight* (kg) & $65(58-72)$ & $62(54-67)$ \\
\hline $\begin{array}{l}\text { Body mass index* } \\
\left(\mathrm{kg} / \mathrm{m}^{2}\right)\end{array}$ & $23.6(20.7-26.2)$ & $23.5(21.5-25.5)$ \\
\hline \multicolumn{3}{|l|}{ Ethnicity } \\
\hline Chinese & $11(37.9)$ & $6(20.7)$ \\
\hline Indian & $13(44.8)$ & $16(55.2)$ \\
\hline Others & $5(17.2)$ & $7(24.1)$ \\
\hline $\begin{array}{l}\text { Maximum temperature } \\
\text { in first } 24 \mathrm{hr}^{*}\left({ }^{\circ} \mathrm{C}\right)\end{array}$ & $37.0(36.8-37.4)$ & $36.9(36.6-37.2)$ \\
\hline $\begin{array}{l}\text { Pre-surgery pain score } \\
\text { at rest* }\end{array}$ & $2(0-3)$ & $1(0-2)$ \\
\hline $\begin{array}{l}\text { Abdominal CT } \\
\text { performed }\end{array}$ & $13(44.8)$ & $13(44.8)$ \\
\hline $\begin{array}{l}\text { Conversion to open } \\
\text { procedure }\end{array}$ & $2(6.9)$ & 0 \\
\hline \multicolumn{3}{|l|}{ Grade of appendicitis } \\
\hline Normal & $3(10.3)$ & $1(3.4)$ \\
\hline Inflamed & $19(65.5)$ & $19(65.5)$ \\
\hline $\begin{array}{l}\text { Inflamed with } \\
\text { complications }\end{array}$ & $4(13.8)$ & $8(27.6)$ \\
\hline $\begin{array}{l}\text { Perforated with } \\
\text { peritonitis }\end{array}$ & $3(10.3)$ & $1(3.4)$ \\
\hline
\end{tabular}

*Data presented as median (interquartile range). СT: computed tomography; TAP: transversus abdominis plane
The mean pain scores at rest and on movement during the first 48 hours following surgery are shown in Fig. 2 and Fig. 3, respectively. Mean pain scores were lower in the TAP group for each time point at rest and on movement. However, these values were not statistically significant, except for values at rest at 36 hours. Pain scores during the first 48 hours following surgery indicated that pain was managed according to standard

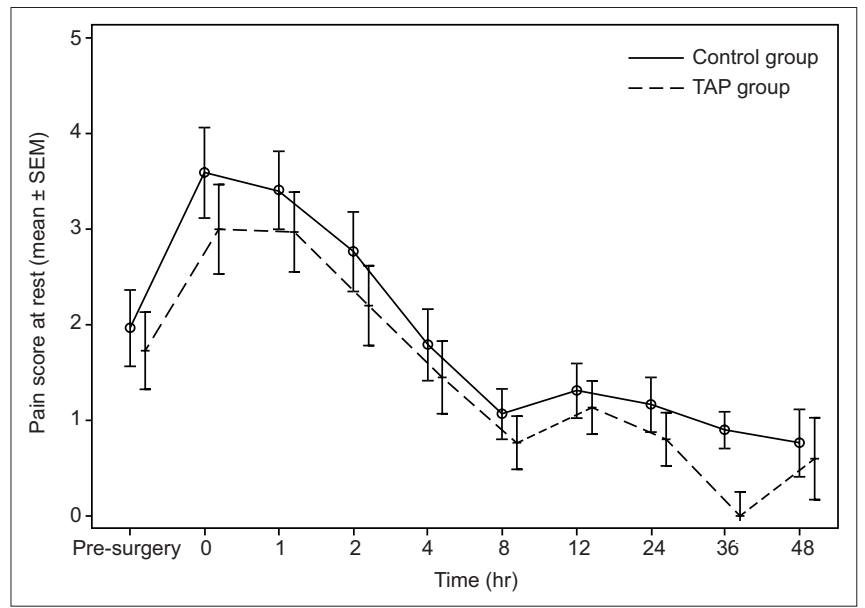

Fig. 2 Pain scores at rest over time for the control and transversus abdominis plane (TAP) groups during the 48-hour study period. SEM: standard error of mean

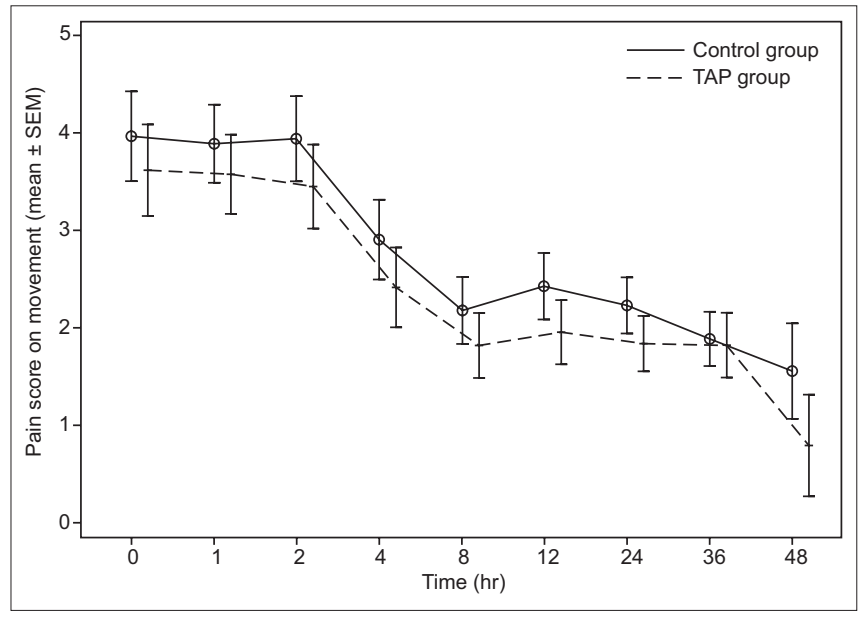

Fig. 3 Pain scores on movement over time for the control and transversus abdominis plane (TAP) groups during the 48-hour study period. SEM: standard error of mean

Table II. Analgesia parameters of the patients according to patient group.

\begin{tabular}{|c|c|c|c|c|}
\hline \multirow[t]{2}{*}{ Parameter } & \multicolumn{2}{|c|}{ No. (\%) } & \multirow[t]{2}{*}{ OR $(95 \% \mathrm{Cl})$} & \multirow[t]{2}{*}{ p-value } \\
\hline & Control $(n=29)$ & TAP $(n=29)$ & & \\
\hline PCA morphine consumption in first $12 \mathrm{hr}^{*}$ (mg) & $11.45 \pm 7.64$ & $9.79 \pm 8.09$ & $1.66(-2.48$ to 5.79$)$ & 0.4264 \\
\hline Total equianalgesic morphine consumption in $24 \mathrm{hr}^{*}(\mathrm{mg})$ & $13.38 \pm 8.72$ & $11.31 \pm 8.66$ & $2.07(-2.48$ to 6.64$)$ & 0.3686 \\
\hline Total dose of intraoperative fentanyl ${ }^{*}(\mathrm{mcg})$ & $160.89 \pm 47.3$ & $128.71 \pm 36.79$ & 32.19 (9.74 to 54.63$)$ & 0.0058 \\
\hline Need for extended PCA (> $18 \mathrm{hr})$ & $2(6.9)$ & $6(20.7)$ & $-0.14(-0.31$ to 0.04$)$ & 0.1277 \\
\hline Need for rescue oral analgesia $(0-24 \mathrm{hr})$ & $9(31.0)$ & $4(13.8)$ & $0.20(-0.02$ to 0.41$)$ & 0.0835 \\
\hline Postoperative nausea and vomiting ( $0-24 \mathrm{hr})$ & $7(24.1)$ & $11(37.9)$ & $-0.14(-0.37$ to 0.1$)$ & 0.3949 \\
\hline High proportion of unsuccessful PCA demands ${ }^{+}$ & $11(39.3)$ & $8(27.6)$ & $0.06(-0.2$ to 0.32$)$ & 0.6568 \\
\hline
\end{tabular}

*Data presented as mean \pm SD. +Defined as > 25\% of unsuccessful PCA bolus demands. Cl: confidence interval; OR: odds ratio; PCA: patient-controlled analgesia; SD: standard deviation; TAP: transversus abdominis plane 
Table III. Perioperative timelines of the patients according to patient group.

\begin{tabular}{|c|c|c|c|c|}
\hline \multirow[t]{2}{*}{ Parameter } & \multicolumn{2}{|c|}{ Mean \pm SD } & \multirow[t]{2}{*}{ OR $(95 \% \mathrm{Cl})$} & \multirow[t]{2}{*}{ p-value } \\
\hline & Control & TAP & & \\
\hline Duration of anaesthesia before start of surgery $(\mathrm{min})$ & $32.18 \pm 9.27$ & $34.07 \pm 9.04$ & $-1.89(-6.8$ to 3.01$)$ & 0.4425 \\
\hline Duration of surgery $(\mathrm{min})$ & $78.64 \pm 35.35$ & $69.64 \pm 19.67$ & $9.00(-6.33$ to 24.33$)$ & 0.2443 \\
\hline Duration of anaesthesia* $(\mathrm{min})$ & $128.90 \pm 37.06$ & $121.62 \pm 20.83$ & 7.28 (-8.54 to 23.09$)$ & 0.3607 \\
\hline Duration in PACU (min) & $72.83 \pm 28.39$ & $57.52 \pm 21.88$ & 15.31 (1.98 to 28.64$)$ & 0.0252 \\
\hline Duration of postop stay to fitness for discharge ${ }^{\dagger}(\mathrm{hr})$ & $39.19 \pm 22.33$ & $32.49 \pm 19.85$ & $6.69(-4.42$ to 17.8$)$ & 0.2328 \\
\hline Duration of total postop stay $(\mathrm{hr})$ & $44.76 \pm 22.40$ & $36.75 \pm 19.95$ & $8.01(-3.14$ to 19.17$)$ & 0.1557 \\
\hline Total duration of hospital stay (day) & $2.68 \pm 1.06$ & $2.29 \pm 0.86$ & $0.39(-0.11$ to 0.90$)$ & 0.1264 \\
\hline
\end{tabular}

*Duration from induction of anaesthesia to arrival in the PACU. +Duration from arrival in the surgical ward to be medically fit for discharge. $\ddagger$ Duration from arrival in the surgical ward to discharge from surgical ward. Cl: confidence interval; OR: odds ratio; PACU: post-anaesthesia care unit; postop: postoperative; SD: standard deviation; TAP: transversus abdominis plane

postoperative pain management goals, which aim for an NRS pain score $\leq 4$.

Table III shows a timeline comparison of the patients' clinical progress, starting from induction of anaesthesia in the operation theatre to the time of discharge from hospital. Hospital discharge times included time from postoperative stay to assessment for fitness for discharge and actual hospital discharge time. PACU stay was significantly shorter in the TAP group compared to the control group $(57.52 \pm 21.88$ minutes vs. $72.83 \pm 28.39$ minutes; $\mathrm{p}=0.0252$ ). In addition, a trend for shorter postoperative stay in the TAP group vs. the control group was observed in terms of the time to fitness for discharge $(32.49 \pm 19.85$ hours vs. $39.19 \pm 22.33$ hours). However, this difference between the two groups was not statistically significant.

\section{DISCUSSION}

Appendicectomy is the most common emergency general surgical procedure performed in developed and developing countries alike. ${ }^{(12,13)}$ Postoperative pain following laparoscopic appendicectomy comprises both parietal and visceral peritoneal components caused by the appendicitis, as well as tissue trauma associated with the surgery and pneumoperitoneum. Pain management following this procedure is important, as either the presence of inadequately treated pain or the occurrence of significant side effects associated with opiate analgesics may prolong surgical recovery. The ability of regional anaesthesia to improve surgical recovery by providing effective postoperative analgesia with reduced opiate analgesic requirements has led to a rise in the use of regional techniques for many surgical procedures. ${ }^{(14)}$

TAP block was first described by Rafi, ${ }^{(15)}$ and the reliability and accuracy of the technique has been enhanced by the advent of ultrasonography-guided needle placement to ensure optimal injection of local anaesthetic solution adjacent to the nerve supply of the abdominal wall. ${ }^{(16)}$ This relatively simple procedure rapidly gained popularity among anaesthetists following the publication of a number of early studies demonstrating improved postoperative analgesia for a range of open abdominal surgical procedures. ${ }^{(5-7,9,17)}$ Given the trend for minimally invasive surgery, with its associated reduction in postoperative pain and faster surgical recovery, there is a need for TAP block to be evaluated specifically for laparoscopic surgical techniques. ${ }^{(18)}$
The present study investigated the postoperative analgesic efficacy of bilateral mid-axillary ultrasonography-guided TAP block in adult patients undergoing laparoscopic appendicectomy. Morphine consumption was the primary endpoint selected to measure postoperative analgesia efficacy, as patients were encouraged to use a self-administering morphine PCA syringe to control the severity of their pain according to a predefined pain score (NRS pain score $\leq 4$ ). For research purposes, this mode of analgesia provides an accurate measure of analgesia requirements following surgery for comparison between the two groups. Both the control and TAP intervention groups received multimodal analgesia regimes with paracetamol and nonsteroidal antiinflammatory drugs, where TAP block was compared to port site local anaesthetic infiltration. Although port site infiltration of local anaesthesia has been shown to have no significant effect on pain or analgesic consumption following laparoscopic procedures, it is routinely practised by a high proportion of surgeons. ${ }^{(19,20)} \mathrm{A}$ high volume of local anaesthetic solution was administered in order to improve the spread of local anaesthetic solution within the TAP tissue plane to maximise analgesic efficacy. Our results showed that TAP block had no significant effect on morphine consumption or pain scores during the first 24 hours following surgery.

Our findings are similar to those of the study by Sandeman et al, ${ }^{(21)}$ where the addition of TAP block to a standard general anaesthetic in a paediatric population did not reduce postoperative analgesic requirements or produce a sustained reduction in postoperative pain scores following laparoscopic appendicectomy. However, our results revealed that TAP block was associated with some benefits in postoperative recovery, including a shorter PACU stay, reduced intraoperative analgesic requirements and a non-significant trend for faster hospital discharge. Therefore, although TAP block is an effective postoperative analgesic therapy for open appendicectomy, ${ }_{,}^{(17,22,23)}$ the benefits of this procedure for laparoscopic appendicectomy are equivocal.

Postoperative pain following laparoscopy may have particular characteristics that are not well controlled by the mid-axillary ultrasonography-guided TAP block. Following laparoscopic gynaecological surgery, a comparison of unilateral ultrasonography-guided TAP block with contralateral port site infiltration failed to show a significant improvement in 
postoperative pain scores when patients were asked to compare pain from both sides of the abdomen. ${ }^{(24)}$ The analgesic effects of TAP block will vary depending on the technique employed; cadaveric and volunteer studies of local anaesthetic spread within the TAP plane have shown important differences between posterior (triangle of Petit), ${ }^{(25)}$ mid-axillary (lateral) ${ }^{(26)}$ and subcostal TAP injection techniques. ${ }^{(27,28)}$ In contrast, a recent meta-analysis of trials investigating TAP block for abdominal laparoscopic surgical procedures demonstrated significant improvements in postoperative pain and reduced opiate requirements. ${ }^{(29)}$ Within this meta-analysis were studies in which the TAP injection technique included subcostal and posterior (triangle of Petit) techniques or the site of injection could not be classified. We postulate that differences in analgesic efficacy between lateral and posterior TAP injection sites may partly explain the lack of measured analgesic effects in our study. The needle injection site with ultrasonography-guided mid-axillary TAP injection deposits local anaesthetic at a lateral point within the TAP plane. Current evidence suggests that a posterior injection site might be more efficacious in terms of postoperative analgesia. ${ }^{(18)}$

The present study was not without limitations, one of which was the absence of any recording of the presence of a failed TAP block. By assigning experienced clinicians experienced in this regional anaesthetic technique to perform the procedure, we aimed to minimise the number of failed or inadequate blocks. Also, achieving a completely blinded TAP block within the limited confines of the operation theatre was not possible for all patients and this may have introduced an element of bias. Although there were no recorded cases of local anaesthesia toxicity in this study, adverse effects due to this complication were difficult to measure, as the block was performed under general anaesthesia. There were also no adverse effects associated with the needle injection into the abdominal wall, indicating that in experienced hands, ultrasonography-guided TAP block is a safe procedure with a low incidence of side effects.

Laparoscopic abdominal surgery encompasses a spectrum of different procedures where postoperative pain arises in response to surgery-specific variables, including the size and site of the surgical wounds, and the extent of intra-abdominal tissue trauma. The ability of TAP block to reduce postoperative pain and improve surgical recovery for laparoscopic surgery requires an area of abdominal wall sensory analgesia that is appropriate for the particular surgical procedure. The reasons for the negative outcome of this study include a significant visceral pain component for postoperative pain as well as the failure of the mid-axillary TAP technique to provide a sufficient area of abdominal wall analgesia. Whether a posterior-directed approach to the TAP plane would achieve clinically significant improvements in postoperative pain for this common procedure has yet to be evaluated.

Future studies investigating postoperative analgesia following laparoscopic appendicectomy should focus on TAP techniques that provide a greater area of abdominal wall analgesia ${ }^{(30,31)}$ or on analgesic therapies targeted toward the visceral pain component. The benefits of TAP block for laparoscopic abdominal procedures appear to be less that of the corresponding open surgical procedures, ${ }^{(21,24,32-34)}$ and presently, the role of this regional anaesthetic procedure for lower abdominal laparoscopic surgery requires further evaluation with larger clinical trials, both to determine the most effective TAP technique and to investigate more precisely its benefits in terms of surgical recovery.

\section{REFERENCES}

1. Bhattacharya K. Kurt Semm: A laparoscopic crusader. J Minim Access Surg 2007; 3:35-6.

2. Li X, Zhang J, Sang L, et al. Laparoscopic versus conventional appendectomy--a meta-analysis of randomized controlled trials. BMC Gastroenterol 2010; 10:129.

3. Pedersen AG, Petersen $O B$, Wara $P$, et al. Randomized clinical trial of laparoscopic versus open appendicectomy. Br J Surg 2001; 88:200-5.

4. Sabbagh C, Brehant O, Dupont $\mathrm{H}$, et al. The feasibility of short-stay laparoscopic appendectomy for acute appendicitis: a prospective cohort study. Surg Endosc 2012; 26:2630-8.

5. Aveline C, Le Hetet H, Le Roux A, et al. Comparison between ultrasound-guided transversus abdominis plane and conventional ilioinguinal/iliohypogastric nerve blocks for day-case open inguinal hernia repair. Br J Anaesth 2011; 106:380-6.

6. Belavy D, Cowlishaw PJ, Howes M, Phillips F. Ultrasound-guided transversus abdominis plane block for analgesia after Caesarean delivery. Br J Anaesth 2009; 103:726-30.

7. Bharti N, Kumar P, Bala I, Gupta V. The efficacy of a novel approach to transversus abdominis plane block for postoperative analgesia after colorectal surgery. Anesth Analg 2011; 112:1504-8.

8. Ra YS, Kim CH, Lee GY, Han JI. The analgesic effect of the ultrasound-guided transverse abdominis plane block after laparoscopic cholecystectomy. Korean J Anesthesiol 2010; 58:362-8.

9. McDonnell JG, O'Donnell B, Curley G, et al. The analgesic efficacy of transversus abdominis plane block after abdominal surgery: a prospective randomized controlled trial. Anesth Analg 2007; 104:193-7.

10. Hebbard P, Fujiwara Y, Shibata Y, Royse C. Ultrasound-guided transversus abdominis plane (TAP) block. Anaesth Intensive Care 2007; 35:616-7.

11. Gerbershagen HJ, Rothaug J, Kalkman CJ, Meissner W. Determination of moderate-to-severe postoperative pain on the numeric rating scale: a cut-off point analysis applying four different methods. Br J Anaesth 2011; 107:619-26.

12. Jawaid M, Raza SM, Alam SN, Manzar S. On-call emergency workload of a general surgical team. J Emerg Trauma Shock 2009; 2:15-8.

13. National Center for Health Statistics. Health, United States, 2013: With Special Feature on Prescription Drugs. Hyattsville, MD, 2014 [online]. Available at: http://www.cdc.gov/nchs/data/hus/hus13.pdf\#116. Accessed June 15, 2017

14. Carli F, Kehlet H, Baldini G, et al. Evidence basis for regional anesthesia in multidisciplinary fast-track surgical care pathways. Reg Anesth Pain Med 2011; 36:63-72.

15. Rafi AN. Abdominal field block: a new approach via the lumbar triangle. Anaesthesia 2001; 56:1024-6.

16. Walter EJ, Smith P, Albertyn R, Uncles DR. Ultrasound imaging for transversus abdominis blocks. Anaesthesia 2008; 63:211.

17. Niraj G, Searle A, Mathews M, et al. Analgesic efficacy of ultrasound-guided transversus abdominis plane block in patients undergoing open appendicectomy. Br J Anaesth 2009; 103:601-5.

18. Abdallah FW, Chan VW, Brull R. Transversus abdominis plane block: a systematic review. Reg Anesth Pain Med 2012; 37:193-209.

19. Ghezzi F, Cromi A, Bergamini V, et al. Preemptive port site local anesthesia in gynecologic laparoscopy: a randomized, controlled trial. J Minim Invasive Gynecol 2005; 12:210-5.

20. Hilvering B, Draaisma WA, van der Bilt JD, et al. Randomized clinical trial of combined preincisional infiltration and intraperitoneal instillation of levobupivacaine for postoperative pain after laparoscopic cholecystectomy. Br J Surg 2011; 98:784-9.

21. Sandeman DJ, Bennett M, Dilley AV, et al. Ultrasound-guided transversus abdominis plane blocks for laparoscopic appendicectomy in children: a prospective randomized trial. Br J Anaesth 2011; 106:882-6.

22. Shaaban AR. Ultrasound guided transversus abdominis plane block versus local wound infiltration in children undergoing appendectomy: A randomized controlled trial. Egypt J Anaesth 2014; 30:377-82.

23. Cho S, Kim YJ, Kim DY, Chung SS. Postoperative analgesic effects of ultrasoundguided transversus abdominis plane block for open appendectomy. J Korean Surg Soc 2013; 85:128-33.

24. El Hachem L, Small E, Chung P, et al. Randomized controlled double-blind trial of transversus abdominis plane block versus trocar site infiltration in gynecologic laparoscopy. Am J Obstet Gynecol 2015; 212:182.e1-9.

25. McDonnell JG, O'Donnell BD, Farrell T, et al. Transversus abdominis plane 
block: a cadaveric and radiological evaluation. Reg Anesth Pain Med 2007; 32:399-404

26. Tran TM, Ivanusic JJ, Hebbard P, Barrington MJ. Determination of spread of injectate after ultrasound-guided transversus abdominis plane block: a cadaveric study. Br J Anaesth 2009; 102:123-7.

27. Lee TH, Barrington MJ, Tran TM, Wong D, Hebbard PD. Comparison of extent of sensory block following posterior and subcostal approaches to ultrasoundguided transversus abdominis plane block. Anaesth Intensive Care 2010; 38:452-60.

28. Barrington MJ, Ivanusic JJ, Rozen WM, Hebbard P. Spread of injectate after ultrasound-guided subcostal transversus abdominis plane block: a cadaveric study. Anaesthesia 2009; 64:745-50.

29. De Oliveira GS Jr, Castro-Alves LJ, Nader A, Kendall MC, McCarthy RJ. Transversus abdominis plane block to ameliorate postoperative pain outcomes after laparoscopic surgery: a meta-analysis of randomized controlled trials. Anesth Analg 2014; 118:454-63.

30. Niraj G, Kelkar A, Hart E, et al. Comparison of analgesic efficacy of four- quadrant transversus abdominis plane (TAP) block and continuous posterior TAP analgesia with epidural analgesia in patients undergoing laparoscopic colorectal surgery: an open-label, randomised, non-inferiority trial. Anaesthesia 2014; 69:348-55.

31. Børglum J, Maschmann C, Belhage B, Jensen K. Ultrasound-guided bilateral dual transversus abdominis plane block: a new four-point approach. Acta Anaesthesiol Scand 2011; 55:658-63.

32. Walter CJ, Maxwell-Armstrong C, Pinkney TD, et al. A randomised controlled trial of the efficacy of ultrasound-guided transversus abdominis plane (TAP) block in laparoscopic colorectal surgery. Surg Endosc 2013; 27:2366-72.

33. Kane SM, Garcia-Tomas V, Alejandro-Rodriguez M, Astley B, Pollard RR. Randomized trial of transversus abdominis plane block at total laparoscopic hysterectomy: effect of regional analgesia on quality of recovery. Am J Obstet Gynecol 2012; 419:e1-5.

34. De Oliveira GS, Milad MP, Fitzgerald P, Rahmani R, McCarthy RJ. Transversus abdominis plane infiltration and quality of recovery after laparoscopic hysterectomy: a randomized controlled trial. Obstet Gynecol 2011; 118:1230-7. 